\title{
Stability analysis of milling with variable pitch tools considering the variable cutting force coefficients with spindle speed
}

\author{
Gang $\mathrm{Jin}^{1, \mathrm{a}}$, Zhanjie $\mathrm{Li}^{1, \mathrm{~b}}$, Jianxin $\mathrm{Han}^{1, \mathrm{c}}$ and $\mathrm{Hua}_{\mathrm{Li}} \mathrm{i}^{2, \mathrm{~d}}$ \\ ${ }^{1}$ Tianjin Key Laboratory of High Speed Cutting and Precision Machining, Tianjin University of \\ Technology and Education, Tianjin, China \\ 2 Tianjin Jinhang Institute of Technical Physical, Tianjin, China) \\ ajgang625802@163.com, 'bmtydhghd@126.com, 'chanjianxin@tju.edu.cn, ${ }^{\mathrm{d}}$ lihua040301@163.com,
}

Keywords: Variable cutting force coefficients; Milling; Chatter; Variable pitch

\begin{abstract}
The combination of increased strain rate and thermal softening of the workpiece at higher cutting speeds will cause the change of cutting force coefficients. In this paper, a vibration model for milling process, which can consider the variable cutting force coefficients (VCFC) with the spindle speed and the variable pitch angle was proposed after some experimental investigations which mainly pay attention to the connection between spindle speed and cutting force coefficients. Then, an updated semi-discretization method is used to predict the stability of such system. The method is also applied to examine the stability trend for different radial immersion ratios and speed regions. Results show that VCFC has great effect on the stability lobes, especially for higher-speed region and smaller radial immersion ratios, whether milling process with uniform or variable pitch angle.
\end{abstract}

\section{Introduction}

High material removal rates often cannot be achieved in practice due to the inherent instability of a cutting process known as chatter. The most powerful source of chatter is regeneration. The approach to gain stability lobe diagram (SLD) through analytical or time-domain solutions is the most established method for predicting and avoiding regenerative chatter [1-9]. For example, the zeroth-order approximation (ZOA) method [1], the semi-discretization method (SDM)[2] and full-discretization method[3], etc. In addition, some methods focused on disrupting the regenerative effect to enlarge the stable zone of the SLD. Sims [4], Dombovari and Stepan [5] and Jin et al [6,7] examine the effect of the tool geometries on the stability trends for variable-helix milling.

It is worth noting that the above works were conducted under the assumption that cutting force coefficients, which are building and linking the relationship between fundamental milling force components and the cut chip area, are constant and treated as static variation in general simulations.However, the milling force coefficients will change with the variation of spindle speed practically. Based on identification of dynamic cutting force coefficients dependent on cutting speed, tool wear and so on, Altintas et al. [8] proposed a model to accurately predict stability at low speeds. At higher cutting speeds, it is known that the combination of increased strain rate and thermal softening of the workpiece will cause the change of cutting force coefficients [9].

The focus of the current manuscript is to investigate the stability trend caused by VCFC under milling with uniform or variable pitch cutter. The work of this paper is organized as follows. Firstly, a vibration model for milling process, which can consider the variation of pitch angle and VCFC with spindle speed was proposed after some works such as the experiments and the curve fitting about VCFC were carried out. Then, an updated SD algorithm in Ref. [6] was used to predict the stability of such system. The next section systematically describes the investigation of the effect of VCFC on chatter stability. The final section describes the conclusions from this work. 


\section{Experimental investigation on VCFC with spindle speed}

Experiments about VCFC. A milling experimental scheme for a range of feed per tooth values ( 0.02 to $0.10 \mathrm{~mm} /$ tooth in steps of $0.02 \mathrm{~mm} /$ tooth) at five different spindle speed values (2000 to $22000 \mathrm{rev} / \mathrm{min}$ in steps of $5000 \mathrm{rev} / \mathrm{min}$ ), is set down to capture the connection between spindle speed and cutting force coefficients, and the details are presented in Table 1 , where $\Omega$ is spindle speed, $a_{p}$ and $a_{e}$ are respectively the axial and radial depth of cut, $V$ is the feed rate and $f_{t}$ is the feed per tooth.

The cutting force coefficient tests were conducted on a DMC $75 \mathrm{~V}$ linear five-axis high-speed machining center (28000rpm spindle). A carbide end mill with $R=12 \mathrm{~mm}, N=4$ was mounted on an EPB-Monobloc HSK-A63 tool holder. The workpiece material was a block of aluminum alloy 6061-T651 with dimensions of $120 \times 100 \times 70(\mathrm{~mm})$ clamped on the Kistler dynamometer 9257B, which was adopted to measure the cutting force and was confined to the worktable.

Table 1: cutting tests to obtain cutting force coefficients at different spindle speed

\begin{tabular}{ccccccc}
\hline Group & $\begin{array}{c}\text { Cutting } \\
\text { number }\end{array}$ & $\begin{array}{c}\Omega \\
(\mathrm{rpm})\end{array}$ & $\begin{array}{c}a_{p} \\
(\mathrm{~mm})\end{array}$ & $\begin{array}{c}a_{e} \\
(\mathrm{~mm})\end{array}$ & $\begin{array}{c}V \\
(\mathrm{~mm} / \mathrm{min})\end{array}$ & $\begin{array}{c}f_{t} \\
(\mathrm{~mm} / \text { tooth })\end{array}$ \\
\hline \multirow{4}{*}{$\mathrm{A}$} & 1 & 2000 & 0.5 & 12 & 160 & 0.02 \\
& 2 & 2000 & 0.5 & 12 & 320 & 0.04 \\
& 3 & 2000 & 0.5 & 12 & 480 & 0.06 \\
& 4 & 2000 & 0.5 & 12 & 640 & 0.08 \\
& 5 & 2000 & 0.5 & 12 & 800 & 0.10 \\
$\mathrm{~B}$ & 1 & 7000 & 0.5 & 12 & 560 & 0.02 \\
& 2 & 7000 & 0.5 & 12 & 1120 & 0.04 \\
& 3 & 7000 & 0.5 & 12 & 1680 & 0.06 \\
& 4 & 7000 & 0.5 & 12 & 2240 & 0.08 \\
& 5 & 7000 & 0.5 & 12 & 2800 & 0.10 \\
$\mathrm{C}$ & 1 & 12000 & 0.5 & 12 & 960 & 0.02 \\
& 2 & 12000 & 0.5 & 12 & 1920 & 0.04 \\
& 3 & 12000 & 0.5 & 12 & 2880 & 0.06 \\
& 4 & 12000 & 0.5 & 12 & 3840 & 0.08 \\
& 5 & 12000 & 0.5 & 12 & 4800 & 0.10 \\
$\mathrm{D}$ & 1 & 17000 & 0.5 & 12 & 1360 & 0.02 \\
& 2 & 17000 & 0.5 & 12 & 2720 & 0.04 \\
& 3 & 17000 & 0.5 & 12 & 4080 & 0.06 \\
& 4 & 17000 & 0.5 & 12 & 5440 & 0.08 \\
& 5 & 17000 & 0.5 & 12 & 6800 & 0.10 \\
$\mathrm{E}$ & 1 & 22000 & 0.5 & 12 & 1760 & 0.02 \\
& 2 & 22000 & 0.5 & 12 & 3520 & 0.04 \\
& 3 & 22000 & 0.5 & 12 & 5280 & 0.06 \\
& 4 & 22000 & 0.5 & 12 & 7040 & 0.08 \\
& 5 & 22000 & 0.5 & 12 & 8800 & 0.10 \\
\hline
\end{tabular}
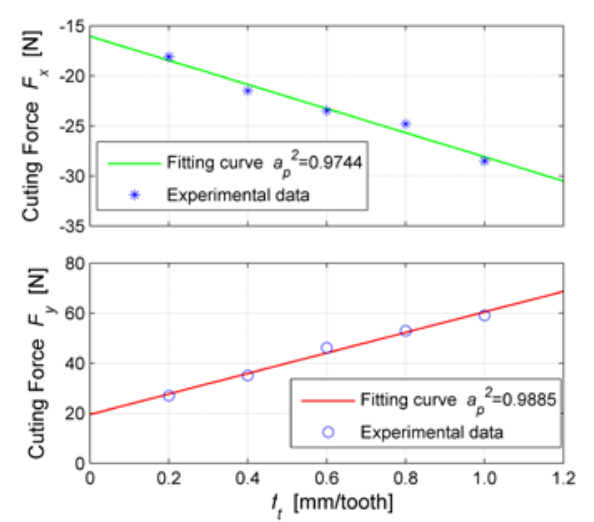

Fig. 1 Mean cutting force versus feed rate 
Here, the method to obtain the cutting force coefficients is the same to the Refs. [1,2], in which the linear regressions performed on the logarithmic variations of mean cutting force values with feed per tooth is developed. Fig. 1 shows the mean force versus feed per tooth plot at $\Omega=2000 \mathrm{rpm}$. Each of the points in Fig. 1 represents the experimental mean cutting force for a particular feed rate, the fitting curves obtained through linear regression from above force values are also supplied and represented by colored line, $a_{p}^{2}$ is the correlation coefficient between experimental and curve fitting data, which is closer to 1 when it is fitting more well. Based on this process, the cutting force coefficients $k_{t c}, k_{r c}, k_{t e}$ and $k_{r e}$ for each of the five spindle speeds were determined, and the results are shown in Table 2.

Table 2: Cutting force coefficients though computation at different spindle speed

\begin{tabular}{ccccrr}
\hline$\Omega(\mathrm{rpm})$ & 2000 & 7000 & 12000 & 17000 & 22000 \\
\hline$k_{\mathrm{tc}}\left(\mathrm{N} / \mathrm{m}^{2}\right)$ & $8.35 \times 10^{8}$ & $7.15 \times 10^{8}$ & $6.68 \times 10^{8}$ & $6.35 \times 10^{8}$ & $6.21 \times 10^{8}$ \\
$k_{r c}\left(\mathrm{~N} / \mathrm{m}^{2}\right)$ & $2.45 \times 10^{8}$ & $1.35 \times 10^{8}$ & $1.02 \times 10^{8}$ & $8.76 \times 10^{7}$ & $7.62 \times 10^{7}$ \\
$k_{t e}\left(\mathrm{~N} / \mathrm{m}^{2}\right)$ & $2.88 \times 10^{4}$ & $2.10 \times 10^{4}$ & $1.82 \times 10^{4}$ & $1.71 \times 10^{4}$ & $1.65 \times 10^{4}$ \\
$k_{r e}\left(\mathrm{~N} / \mathrm{m}^{2}\right)$ & $2.64 \times 10^{4}$ & $2.21 \times 10^{4}$ & $2.01 \times 10^{4}$ & $1.65 \times 10^{4}$ & $1.54 \times 10^{4}$ \\
\hline
\end{tabular}

Formula of VCFC. Nonlinear method LSQNONLIN and function $y(x)=a_{i 0}+b_{i 0} x^{c_{i 0}}$ $(i=t, r)$ are adopted to fit the values of the scattered data points of the cutting force coefficients in Table 2 (only curve fittings of $k_{t c}$ and $k_{r c}$ are carried out), the fitting formula are:

$$
\begin{aligned}
& k_{t c}(\Omega)=1+2.1526 \times 10^{9} \times \Omega^{-0.1249} \\
& k_{r c}(\Omega)=-7.8213 \times 10^{6}+8.2933 \times 10^{9} \times \Omega^{-0.4592}
\end{aligned}
$$

where $\Omega$ is the spindle speed.

The related plot results corresponding to Eq. (1) are shown in Fig. 2 for comparison. As can be seen from Fig. 2, a decrease occurs whether for the tangential or normal linearized cutting force coefficients as the spindle speed is increased. This means the effect of spindle speed on CFC is obvious, and is relatively different for various cutting region, which is more severe in lower regions than that in high one.

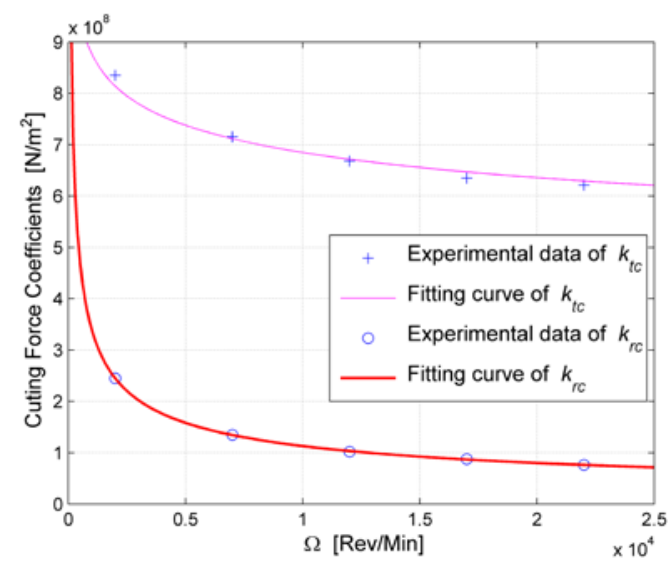

Fig. 2 the relationship between cutting force coefficients and spindle speed

\section{Model formulation}

The dynamic model considered here is shown in Fig. 3 where the cross-sectional view of 2 degree of freedom milling process is supplied. A cutter with radius $R$ and $N$ equally or unequally spaced teeth rotating at a constant velocity $\Omega$ (rev/min) is assumed to have flexibility in two orthogonal directions (x, y), if the effect of $\Omega$ on the CFC is considered simultaneously, the mathematical equation of these systems can be synthetically expressed as: 


$$
\boldsymbol{M} \ddot{\boldsymbol{u}}(t)+\boldsymbol{C} \dot{\boldsymbol{u}}(t)+\boldsymbol{K} \boldsymbol{u}(t)=a_{p} \sum_{j=1}^{N} \boldsymbol{H}_{j}(t, \Omega)\left(\boldsymbol{u}\left(t-\tau_{j}\right)-\boldsymbol{u}(t)\right)
$$

where vector $\boldsymbol{u}(t)=[x(t), y(t)]^{T}$ contains the displacement of the tool tip in the $\mathrm{x}$ and $\mathrm{y}$ directions whereas $\boldsymbol{M}, \boldsymbol{C}$, and $\boldsymbol{K}$ are the modal mass, damping, and stiffness matrices, respectively. $\tau_{j}$ is the pitch period corresponding to $\psi_{j}$ (the pitch angle between the $j$ th and the $j$-1th teeth). The matrix of the directional cutting force coefficients $\boldsymbol{H}$ are indirect affected by the spindle speed $\Omega$ (based on the analysis in section 2) and can be written as:

with

$$
\boldsymbol{H}_{j}(t, \Omega)=\left[\begin{array}{ll}
h_{x x, j}(t, \Omega) & h_{x y, j}(t, \Omega) \\
h_{y x, j}(t, \Omega) & h_{y y, j}(t, \Omega)
\end{array}\right]
$$

$$
\begin{aligned}
& h_{x x, j}(t, \Omega)=g\left(\phi_{j}(t)\right) \sin \left(\phi_{j}(t)\right)\left(k_{t c}(\Omega) \cos \left(\phi_{j}(t)\right)+k_{r c}(\Omega) \sin \left(\phi_{j}(t)\right)\right) \\
& h_{x y, j}(t, \Omega)=g\left(\phi_{j}(t)\right) \cos \left(\phi_{j}(t)\right)\left(k_{t c}(\Omega) \cos \left(\phi_{j}(t)\right)+k_{r c}(\Omega) \sin \left(\phi_{j}(t)\right)\right) \\
& h_{y x, j}(t, \Omega)=g\left(\phi_{j}(t)\right) \sin \left(\phi_{j}(t)\right)\left(-k_{t c}(\Omega) \sin \left(\phi_{j}(t)\right)+k_{r c}(\Omega) \cos \left(\phi_{j}(t)\right)\right) \\
& h_{y y, j}(t, \Omega)=g\left(\phi_{j}(t)\right) \cos \left(\phi_{j}(t)\right)\left(-k_{t c}(\Omega) \sin \left(\phi_{j}(t)\right)+k_{r c}(\Omega) \cos \left(\phi_{j}(t)\right)\right)
\end{aligned}
$$

where $g\left(\phi_{j}(t)\right)$ is a window function which is used to indicate whether the tooth is in or out of the cut. $\phi_{j}(t)$ defines the angular position of tooth $j$ at time $t$ and can be expressed as:

$$
\phi_{j}(t)=\left\{\begin{array}{c}
\frac{2 \pi \Omega}{60} t, \quad \text { if } j=1 \\
\frac{2 \pi \Omega}{60} t+\sum_{i=2}^{j} \psi_{i}, \text { if } 1<j \leq N
\end{array}\right.
$$

The related algorithm to solve above Eq. (2) will not be shown anymore because of the limitation of space. For more details, one can refer to Ref. [6].

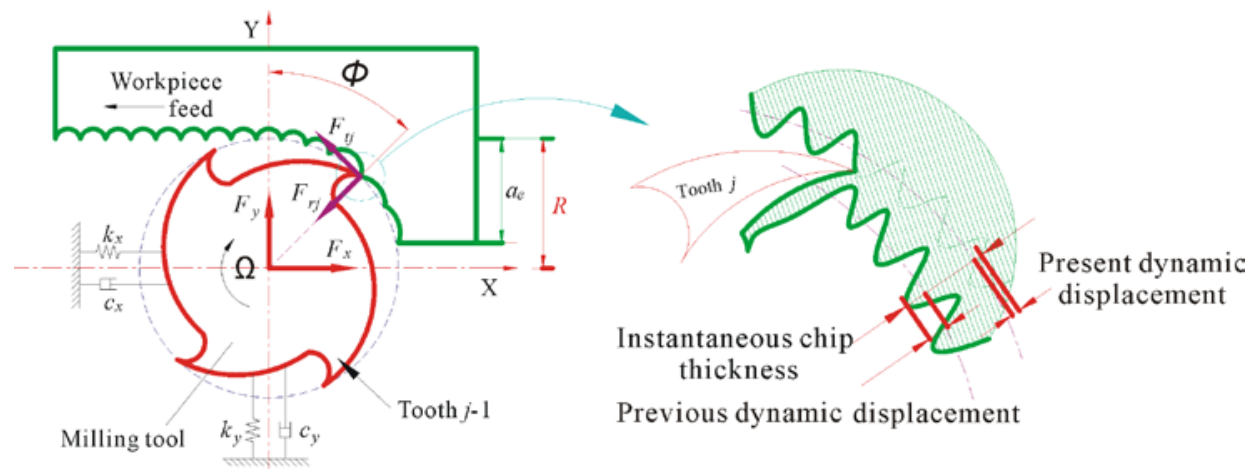

Fig. 3 Chatter modeling for milling

\section{Stability prediction}

To see the effect of VCFC with spindle speed on stability lobes, the simulations are firstly conducted on the base of the experimental parameters referred to the works of Altintas et al.[1]. Noted that for the case of VCFC, the original $k_{t c}=679 \mathrm{Mpa}$ and $k_{r c}=256 \mathrm{Mpa}$ in case of CCFC are assumed to measure at speed 2000rpm, and thus the parameters in Eq. (1) should be changed to $a_{t 0}=-1.36 \times 10^{8}$ and $a_{r 0}=-2.93 \times 10^{6}$ to make the curve of VCFC under the circumstances keeping an identical trend with that in Fig. 2.

The simulation results of SLD for milling process with uniform and variable pitch cutter are plotted in Fig. 4. It can be seen that once the VCFC are considered, apparent deviations occur between the two stability charts. Obviously, the case of VCFC can obtain a higher stability chart, and 
this trend seems more significant following the increasing of spindle speed. The reason of this may be that the decrease of cutting force, caused by the variation of VCFC with spindle speed lowers the possibilities of milling chatter. Besides, the above phenomenon also occurs in the case of variable pitch milling as shown in Fig. 4(b). These indicate that VCFC has obvious influence on the stability boundaries for milling process, whether with uniform or with variable pitch cutter.
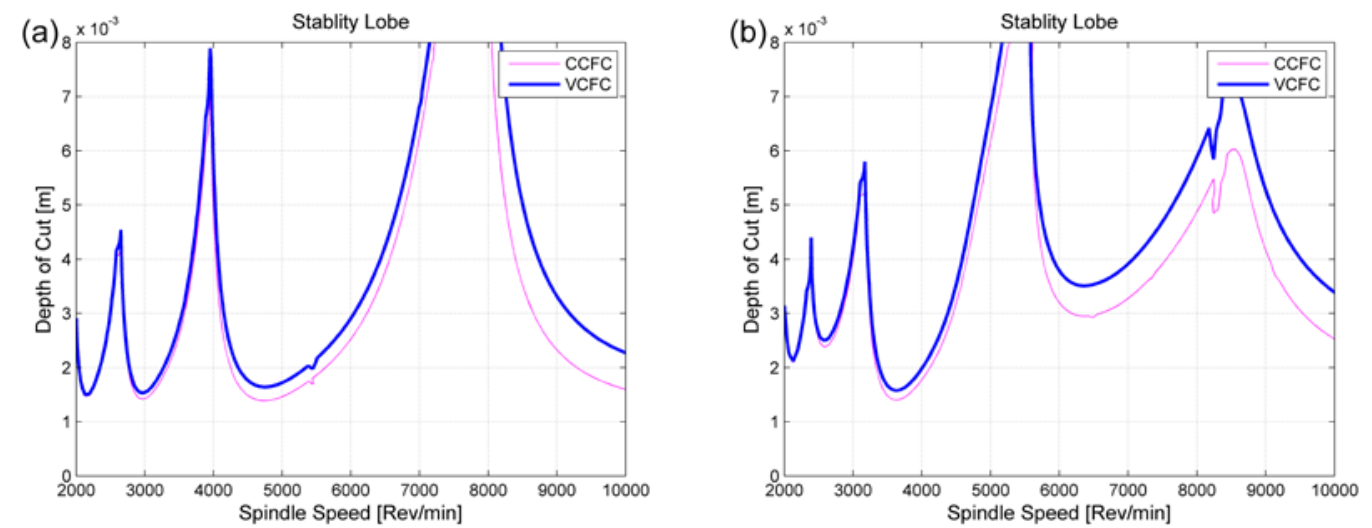

Fig. 4 Stability lobes for milling process considering CCFC and VCFC using different cutters. (a) uniform pitch cutter; (b) variable pitch cutter.
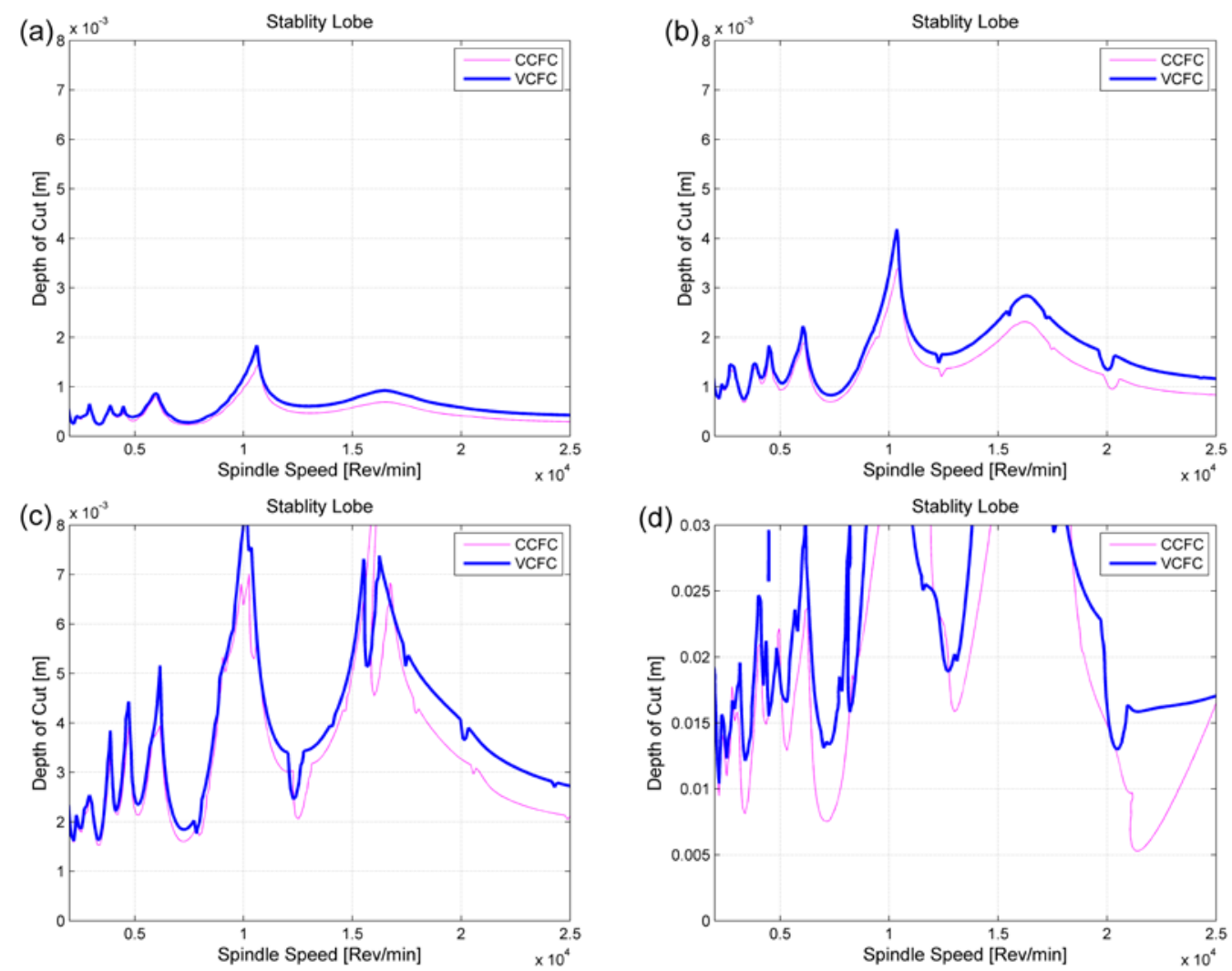

Fig. 5 Stability lobes for milling process with variable pitch cutter considering CCFC and VCFC under different radial immersion ratios. (a) 1 ; (b) 0.5 ; (c) 0.25 ; (d) 0.05 .

To illustrate the performance of radial immersion ratios on dynamic behaviour of the milling system with CCFC and VCFC, the effects are numerically studied for down milling operations with variable pitch cutter at four different radial immersion ratios, i.e., 100\%, 50\%, 25\% and 5\% radial immersions, and related stability charts are shown in Figs. 5. It can be seen from Fig. 5 that for each of the four radial immersion ratios, the range of stable axial depth of cut for the milling with VCFC is larger than that for the corresponding on with CCFC, comparing the stability lobes for the VCFC 
milling process with those obtained for the CCFC milling process. This demonstrates that VCFC has important influence on milling stability for every radial immersion ratios.

However, comparing the simulation results from Fig. 5(a) to 5(d), it can be seen that the deviations between stability charts respectively considering CCFC and VCFC is bigger with regard to the smaller radial immersion ratios. This indicts that the VCFC have more evident effect on stability under circumstance of lower radial immersion ratios. This means that the considerations of VCFC can make much more sense in low radial immersion ratios than that in high case for high-speed milling process.

For different regions of cutting speed, it can be seen from any graph in Fig. 5 that with the rise of spindle speed, the deviations of stability charts corresponding to cases of CCFC and VCFC become bigger. This means that the considerations of VCFC can make much more sense in high-speed milling process than that in case of lower-speed one.

\section{Conclusion}

In this paper, an analytical method, which can consider the variable cutting force coefficients with the spindle speed and the variable pitch angle, is presented for predicting the stability lobes of milling process with uniform and variable pitch cutter. Research attention is paid on the influence of VCFC on the stability trends under different radial immersion for uniform or variable pitch cutter milling. The following conclusions are achieved:

1. VCFC has obvious influence on the stability boundaries for milling process, whether with uniform or with variable pitch cutter.

2. The considerations of VCFC can make much more sense in high-speed milling process than that in case of lower-speed one.

3. VCFC has important influence on milling stability for every radial immersion ratios, especially under circumstance of the lower radial immersion ratios.

\section{Acknowledgements}

This work was supported by the National Natural Science Foundation of China (grant no. 51405343), Tianjin Research Program of Application Foundation and Advanced Technology (15JCQNJC05000), Innovation Team Training Plan of Tianjin Universities and colleges (TD12-5043), the cultivation plan for young and key creative talents in Tianjin (KC14-07)"

\section{References}

[1] Y. Altintas, S. Engin and E. Budak: J. Manuf. Sci. Eng Vol. 121(1999), p. 173-178.

[2] T. Insperger, B. Mann, G. Stepan, et al: Int. J. Mach. Tools Manuf Vol. 43(2003), p. 25-34.

[3] Y. Ding, L. Zhu, X. Zhang, et al: Int. J. Mach. Tools Manuf Vol. 50(2010), p.502-509.

[4] N. Sims, B. Mann and S. Huyanan: J. SOUND VIB. Vol. 317(2008), p.664-686.

[5] Z. Dombovari and G. Stepan: J. Manuf. Sci. Eng. Vol. 314(2012), p. 051015-1-6.

[6] G. Jin, Q. Zhang, H. Qi et al: P I MECH ENG C- JMC. Vol. 228(2014), p. 2702-2710.

[7] G. Jin, Q. Zhang, S. Hao et al: P I MECH ENG C- JMC. Vol. 228(2014), p. 281-293.

[8] Y. Altintas, M. Eynian, H. Onozuka: CIRP ANN-MANUF TECHN, Vol. 57(2008), p. 371-374.

[9] K. Powell. Ph.D. Dissertation. University of Florida. 2008. 